\title{
Risk Factors of Ischemic Lesions Related to Cerebral Angiography and Neuro-interventional Procedures
}

\author{
Masayuki SATO, ${ }^{1}$ Yasunobu NAKAI, ${ }^{1}$ Hideo TSURUSHIMA, ${ }^{1}$ \\ Masanari SHIIGAI, ${ }^{2}$ Tomohiko MASUMOTO, ${ }^{2}$ and Akira MATSUMURA ${ }^{1}$ \\ Departments of ${ }^{1}$ Neurosurgery, and ${ }^{2}$ Intervention and Radiology, \\ Faculty of Medicine, University of Tsukuba, Tsukuba, Ibaraki
}

\begin{abstract}
Embolic stroke is not a rare complication of cerebral angiography. The risk factors for incidental embolism after cerebral angiography were retrospectively examined using diffusion-weighted magnetic resonance imaging (DWI) in 180 patients who underwent 247 angiography procedures, consisting of 174 diagnostic angiography and 73 interventional procedures, and magnetic resonance imaging including DWI within 72 hours after angiography. The two neuroradiologists in our hospital detected embolism after cerebral angiography as high-intensity lesions (HIL) on DWI. The relationships between HIL on DWI and procedural factors were evaluated. DWI after cerebral angiography revealed HIL related to the procedure in 72 of 247 angiographies. In all procedures, age $(p<0.01)$, past history of cerebral infarction $(p<0.05)$, anti-platelet therapy $(p<0.05)$, neuro-intervention $(p<0.01)$, and total amount of contrast medium (odds ratio [OR] $2.125,95 \%$ confidence interval [CI] 1.045-4.321) were significantly correlated with HIL. In diagnostic angiography, the performance of the procedure by a resident operator (OR 2.526, 95\% CI 1.214-5.254) was significantly correlated with HIL. Age, past history of cerebral infarction, and previous anti-platelet therapy determined the risk of atherosclerotic changes in patients. The neuro-intervention and total amount of contrast medium used could predict the risk of time limitations for angiography. Resident operator is also a risk factor. This study demonstrates the importance of improving the risk of time limitations for angiography and the risk due to operator inexperience. Further training of residents may be needed to reduce the occurrence of embolic complications.
\end{abstract}

Key words: cerebral angiography, diffusion-weighted magnetic resonance imaging, cerebral embolism, complication

\section{Introduction}

Neuroimaging techniques, such as magnetic resonance (MR) imaging and multi-detector computed tomography, have been improving in recent years. However, cerebral angiography is still an important tool for examining cerebral vascular diseases and performing neuro-interventional procedures. This technique carries a small but defined risk for neurological complications with incidental embolism. Previous reports have indicated that incidental embolism is not rare, and past history of vasculopathy, amount of contrast medium used, fluoroscopy time, use of additional catheters, procedures of the occlusion test, and performance of endovascular surgery for cerebrovascular disease are

Received November 1, 2012; Accepted December 20, 2012 risk factors for embolism. $1,4,5,8,9,11,13,16,17,20,21)$ Angiography of the coronary arteries also carries a risk for cerebral embolism due to the duration of the procedure.4,15) Diffusion-weighted MR imaging (DWI) allows for the most sensitive early detection of cerebral ischemia within minutes of onset. ${ }^{22}$ The present study tried to examine the risk factors for cerebral embolism after cerebral angiography and neuro-interventional procedures using DWI.

\section{Methods}

From October 2008 to March 2010, 180 patients (73 men and 107 women, mean age 56.0 years [range 5-92 years]) underwent 247 cerebral angiography procedures in our university hospital. These patients were enrolled in this study retrospectively. Of these patients, 174 belonged to the diagnostic angiography group, and 73 belonged to the neuro-inter- 
Table 1 Comparison of baseline clinical characteristics and subsequent disease diagnosis

\begin{tabular}{|c|c|c|c|c|}
\hline & \multirow{2}{*}{ All cases $(n=247)$} & \multicolumn{2}{|c|}{ DWI } & \multirow{2}{*}{$\mathrm{p}$ Value } \\
\hline & & Yes $(n=72)$ & No $(\mathrm{n}=175)$ & \\
\hline \multicolumn{5}{|l|}{ Clinical characteristic: } \\
\hline Age $^{*}$, yrs & $56.6 \pm 19.8$ & $63.1 \pm 17.3$ & $54.3 \pm 20.4$ & $<0.05$ \\
\hline Female $(\%)$ & $153(61.9)$ & $43(59.8)$ & $110(62.9)$ & NS \\
\hline Myocardial infarction (\%) & $20(8.1)$ & $10(13.9)$ & $10(5.7)$ & NS \\
\hline Cerebral infarction (\%) & $66(26.7)$ & $26(36.1)$ & $40(22.9)$ & $<0.05$ \\
\hline Diabetes (\%) & 40 (16.2) & $14(19.4)$ & $26(14.9)$ & NS \\
\hline Hypertension (\%) & $124(50.2)$ & $42(58.3)$ & $82(46.9)$ & NS \\
\hline Hypercholesterolemia (\%) & 48 (19.4) & $10(13.9)$ & $38(21.7)$ & NS \\
\hline Uric acid abnormality (\%) & $3(1.2)$ & $0(0)$ & $3(1.7)$ & NS \\
\hline Chronic renal failure (\%) & $9(3.6)$ & $3(4.2)$ & $6(3.4)$ & NS \\
\hline Obesity (\%) & $8(3.2)$ & $1(1.4)$ & $7(4.0)$ & NS \\
\hline Smoking (\%) & $70(28.3)$ & $25(34.7)$ & $45(25.7)$ & NS \\
\hline Anti-platelet therapy (\%) & $84(34.0)$ & $32(44.4)$ & $52(29.7)$ & $<0.05$ \\
\hline \multicolumn{5}{|l|}{ Subsequent diagnosis: } \\
\hline Unruptured aneurysm (\%) & $61(24.7)$ & $19(26.4)$ & $42(24.0)$ & NS \\
\hline Arteriovenous malformation (\%) & $30(12.1)$ & $6(8.3)$ & $24(13.7)$ & NS \\
\hline Cervical artery stenosis (\%) & $58(23.5)$ & $19(26.4)$ & $29(16.6)$ & NS \\
\hline Dural arteriovenous fistula (\%) & $21(8.5)$ & $7(9.7)$ & $14(8.0)$ & NS \\
\hline Infarction (\%) & $8(3.2)$ & $1(1.4)$ & $7(4.0)$ & NS \\
\hline Moyamoya disease (\%) & $16(6.5)$ & $5(6.9)$ & $11(6.3)$ & NS \\
\hline Tumor $(\%)$ & $34(13.8)$ & $7(9.7)$ & $27(15.4)$ & NS \\
\hline Others (\%) & $29(11.7)$ & $8(11.1)$ & $21(12.0)$ & NS \\
\hline
\end{tabular}

*Values are mean \pm standard deviation. DWI: diffusion-weighted magnetic resonance imaging, NS: not significant.

ventional procedure group. The patients had the following diagnoses: unruptured cerebral aneurysm (n $=61)$, arteriovenous malformation $(\mathrm{n}=30)$, cervical artery stenosis $(\mathrm{n}=58)$, dural arteriovenous fistula $(\mathrm{n}=21)$, infarction $(\mathrm{n}=8)$, moyamoya disease $(\mathrm{n}=16)$, brain tumor $(\mathrm{n}=34)$, and others $(\mathrm{n}=$ 29) (Table 1). Informed consent was obtained from each subject. We identified a subgroup of patients with vascular risk factors, listed as follows: age, sex, previous myocardial infarction and cerebral infarction, diabetes, hypertension, hypercholesterolemia, uric acid abnormality, chronic renal failure, obesity (body mass index $\geq 30$ ), smoking, and previous antiplatelet therapy. These risk factors included both well controlled and not controlled cases. The Institutional Ethics Committee at our university hospital approved this study.

Cerebral angiography was performed through a trans-femoral artery approach $(n=229)$ or a transradial artery approach $(\mathrm{n}=18)$. Diagnostic angiography was performed with a $4 \mathrm{Fr}$ catheter and a standard guide wire (Radifocus, 0.035 inch diameter; Terumo, Tokyo). Neuro-interventional procedures were performed with appropriately sized catheters and guide wires for each case. We used nonionic contrast medium (OMNIPAQUE 300;
Daiichi Sankyo, Tokyo) in all cases. During the neuro-interventional procedure, the catheters were continuously flushed with heparin and additional saline unless a guide wire was used. The flushing system consisted of a high-pressure infusion system with a drop catheter (permanent pressure $>300 \mathrm{mmHg}$ ), which was permanently attached to the catheter.

The following factors were recorded in each case: total procedure time; total amount of contrast medium used; total number of examinations; whether an additional catheter was needed; whether the patient underwent three-dimensional rotation angiography; and the anti-coagulation therapy used during angiography (Table 2). In our university hospital, neurosurgeons and neuroradiologists always perform angiography together and discuss the findings at the time. Cerebral angiography was performed by more than two operators who are resident operators with senior operators or two senior operators.

The senior operators were two neurosurgeons or one neuroradiologist with licenses from the Japanese Society for Neuroendovascular Therapy or the Japanese Radiological Society, which guarantees that the operator has performed more than 300 cerebral angiographies in the last 5 years. The resident operators were physicians who had held medi- 
Table 2 Comparison of three magnetic resonance (MR) imaging scanners and the procedural factor

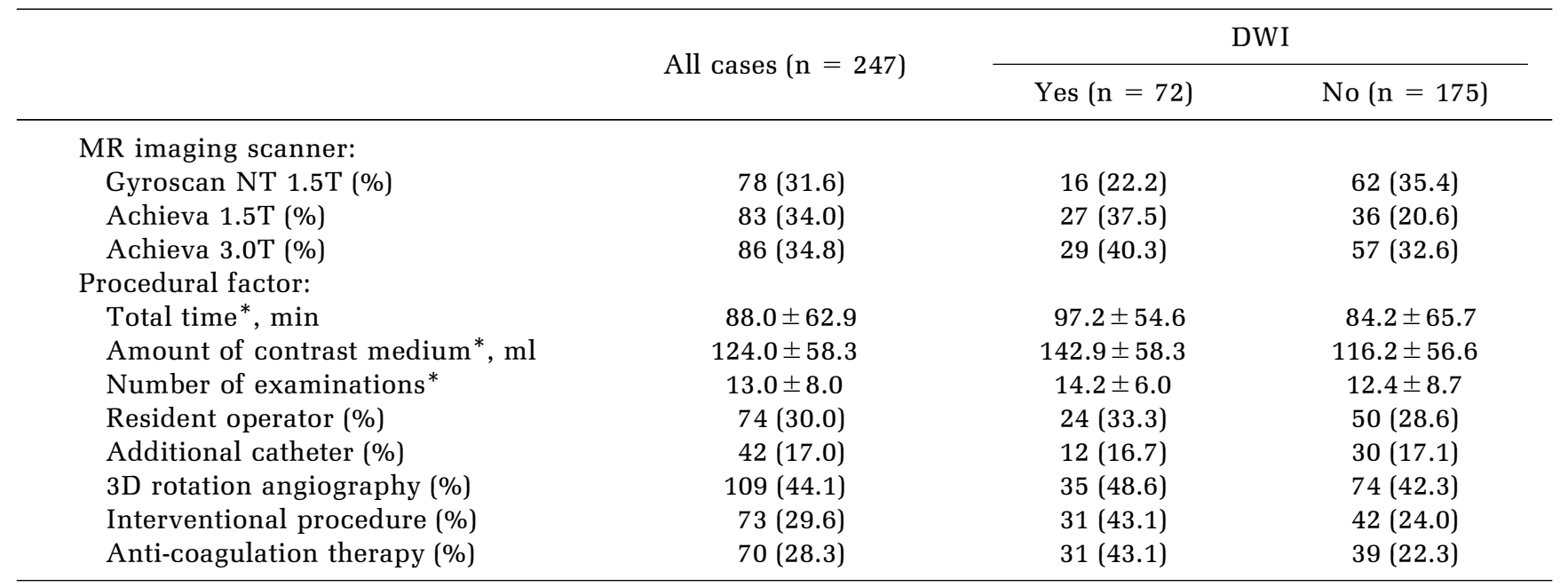

*Values are mean \pm standard deviation. 3D: three-dimensional, DWI: diffusion-weighted magnetic resonance imaging.

cal licenses for up to 6 years and had performed fewer than 100 diagnostic cerebral angiographies in their career.

All patients underwent MR imaging including DWI within 72 hours after angiography using three MR imaging scanners, as follows: Gyroscan NT 1.5T (Phillips Medical Systems, Einthoven, the Netherlands), Achieva 1.5T (Phillips Medical Systems), and Achieva 3.0T (Phillips Medical Systems). DWI was obtained using a single-shot echo-planar spin-echo sequence. The protocol of each scanner was as follows: Gyroscan NT 1.5T, repetition time (TR) = $4070.2 \mathrm{msec}$, echo time $(\mathrm{TE})=88.0 \mathrm{msec}$, slice thickness $(\mathrm{ST})=5.0 \mathrm{~mm}$, matrix $=256 \times 256, \mathrm{~b}=$ $1000 \mathrm{sec} / \mathrm{mm}^{2}$; Achieva $1.5 \mathrm{~T}$, TR $=3500 \mathrm{msec}$, TE $=67.1 \mathrm{msec}, \mathrm{ST}=5.0 \mathrm{~mm}$, matrix $=256 \times 256, \mathrm{~b}$ $=1000 \mathrm{sec} / \mathrm{mm}^{2} ;$ and Achieva $3.0 \mathrm{~T}, \mathrm{TR}=5000$ msec, $\mathrm{TE}=57.5 \mathrm{msec}, \mathrm{ST}=5.0 \mathrm{~mm}$, matrix $=288$ $\times 288, b=1000 \mathrm{sec} / \mathrm{mm}^{2}$ ). According to the Acute Stroke Imaging Standardization Group-Japan (ASIST-Japan) for standardization of DWI, the two neuroradiologists in our university hospital set the window width equal to the signal intensity in the b0 images of the normal-appearing thalamus and then set the window level, ${ }^{19)}$ and evaluated the DWI findings after cerebral angiography. The patient distribution was as follows: Gyroscan NT 1.5T (78 cases, 16 DWI positive), Achieva $1.5 \mathrm{~T}$ (83 cases, 27 DWI positive). and Achieva 3.0T (86 cases, 29 DWI positive) (Table 2). We statistically compared the historical differentiation between these three MR imaging scanners. We referred to the recommended protocol of ASIST-Japan and standardized the DWI by setting the window width and window level. The DWI obtained from the three MR imaging scanners showed no significant differences in the background distribution, frequency of HIL on DWI, or operator distribution. The histories of patients and the types of procedures (diagnosis or neuro-intervention) were concealed during the image analysis.

Absence of high-intensity lesions (HIL) on DWI was not confirmed before angiography in all patients. Usually, small HIL on DWI vanish within 2 weeks. We excluded patients who suffered cerebral infarction or transient ischemic attack within 1 month before angiography, ${ }^{3,15)}$ so that we could consider small HIL on DWI to be related to the present angiographic procedure and as indicators of cerebral microembolism.

Continuous variables are expressed as the mean value \pm standard deviation, and comparisons of these variables between groups were performed using the Student's t-test. Categorical variables are expressed as counts and percentage frequencies, and were compared using the chi-square test. A multivariate logistic regression test was used to identify independent predictors of risk for cerebral embolism from procedural factors. A $p$ value of $<0.05$ was considered to be significant. The differences between the MR imaging scanners were analyzed statistically by one-way analysis of variance (ANOVA) and Tukey's post-hoc tests $(\alpha=0.05)$. SPSS ver.19 for Windows (IBM, Armonk, New York, USA) was used for all statistical analyses.

\section{Results}

DWI after cerebral angiography revealed HIL related to the procedure in 72 of 247 angiographies (Fig. 1). The baseline patient clinical characteristics and 

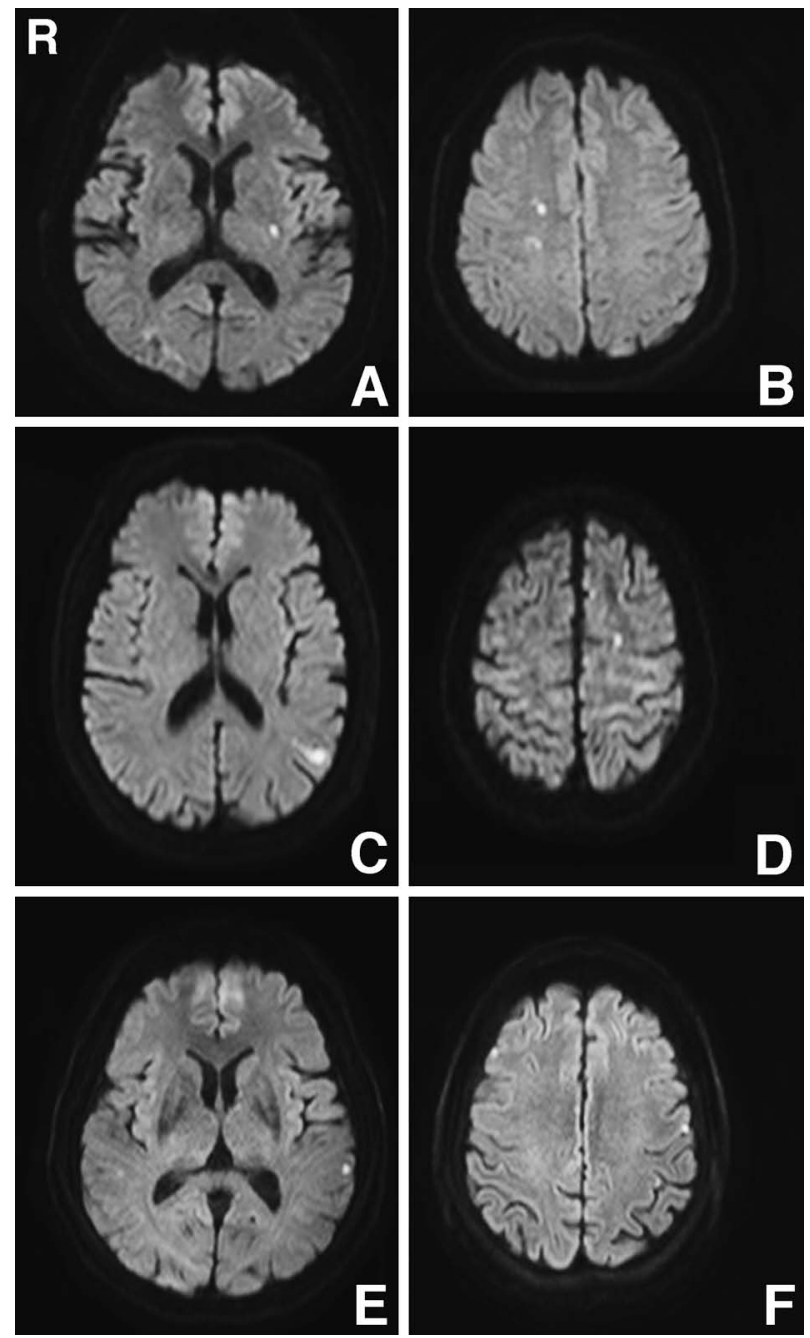

Fig. 1 Typical cases of high intensity lesions on diffusion-weighted magnetic resonance imaging indicating silent embolism after cerebral angiography. A: A 72-year-old man with cervical artery stenosis (Gyroscan NT 1.5T), B: a 32-year-old woman with an unruptured cerebral aneurysm (Gyroscan NT 1.5T), C: a 72-year-old man with an unruptured cerebral aneurysm (Achieva 1.5T), D: a 75-year-old woman with an unruptured cerebral aneurysm (Achieva 1.5T), E: a 65-year-old woman with an unruptured cerebral aneurysm (Achieva 3.0T), F: a 52-year-old woman with an unruptured cerebral aneurysm (Achieva 3.0T).

the diagnosis by angiography are shown in Table 1 . Age ( $p<0.05)$, past history of cerebral infarction ( $p$ $<0.05$ ), and previous anti-platelet therapy ( $p<$ 0.05 ) were significantly different between the two groups. Sex, preceding myocardial infarction, diabetes, hypertension, hypercholesterolemia, uric acid abnormality, chronic renal failure, obesity, and smoking were not significantly different. Six of the 72 patients in the HIL group had symptomatic neu- rological deficits after angiography. Of these, four patients had transient symptoms and two patients had permanent neurological deficit. The frequency of disease diagnosis by angiography was similar in both groups. For procedural factors, univariate analysis showed that total time $\geq 80$ minutes (odds ratio [OR] 2.057, 95\% confidence interval [CI] 1.1673.626 ), amount of contrast medium $\geq 100 \mathrm{ml}$ (OR $2.833,95 \%$ CI $1.539-5.216$ ), $\geq 12$ examinations (OR 2.101, 95\% CI 1.204-3.667), neuro-intervention (OR 2.394, 95\% CI 1.339-4.282), and anti-coagulation therapy (OR 1.804, 95\% CI 1.260-2.581) were risk factors (Table 3). Additionally, multivariate analysis indicated that amount of contrast medium $\geq 100 \mathrm{ml}$ (OR 2.125, 95\% CI 1.045-4.321) was a significant risk factor. The mean procedure duration was $97.2 \pm$ 54.6 minutes in patients with HIL on DWI and 84.2 \pm 65.7 minutes in patients without HIL (Table 2). There was no significant difference in total procedure time.

A senior operator performed most neuro-interventional procedures. Therefore, we analyzed the subgroup receiving only diagnostic cerebral angiography, which represented 174 diagnostic cerebral angiographies. Of these, 41 patients had HIL on DWI. Univariate analysis identified the following risk factors: total amount of contrast medium $\geq 100 \mathrm{ml}$ (OR 2.383, 95\% CI 1.157-4.910), $\geq 10$ examinations (OR 2.054, 95\% CI 1.011-4.174), and performance of the procedure by a resident operator (OR 2.417, 95\% CI 1.185-4.929) (Table 4). Additionally, multivariate analysis indicated that performance of the procedure by a resident operator (OR 2.526, 95\% CI 1.214-5.254) was the only significant risk factor. The mean procedure duration was 64.1 \pm 20.2 minutes in patients with HIL on DWI and $60.0 \pm 21.5$ minutes in patients without HIL. There was no significant difference in the total procedure time. Analysis by one-way ANOVA and Tukey's post-hoc tests revealed no significant difference between the three MR imaging scanners.

\section{Discussion}

Previous articles have reported that the risk of HIL on DWI after cerebral angiography ranges from $9 \%$ to $26 \% .^{1,2,14)}$ The amount of contrast medium used, fluoroscopic time, and use of additional catheters were risk factors for the detection of HIL on DWI after cerebral angiography.1) The results of occlusion tests of the internal carotid arteries were significantly correlated with the appearance of HIL on DWI. ${ }^{14)}$ Additionally, a history of stroke and transient ischemic attack were more highly correlated with neurological complication after angiogra- 
Table 3 Univariate and multivariate analysis of risk factors for all cases

\begin{tabular}{|c|c|c|c|c|c|c|}
\hline & \multicolumn{3}{|c|}{ Univariate analysis } & \multicolumn{3}{|c|}{ Multivariate analysis } \\
\hline & Odds ratio & $95 \%$ CI & $\mathrm{p}$ Value & Odds ratio & $95 \%$ CI & $\mathrm{p}$ Value \\
\hline Total time $\geq 80 \mathrm{~min}$ & 2.057 & $1.167-3.626$ & 0.013 & 0.976 & $0.427-2.232$ & 0.954 \\
\hline Amount of contrast medium $\geq 100 \mathrm{ml}$ & 2.833 & $1.539-5.216$ & 0.001 & 2.125 & $1.045-4.321$ & 0.037 \\
\hline Number of examinations $\geq 12$ & 2.101 & $1.204-3.667$ & 0.009 & 1.273 & $0.662-2.445$ & 0.469 \\
\hline Resident operator & 1.25 & $0.693-2.254$ & 0.458 & & & \\
\hline Additional catheter & 0.967 & $0.467-2.014$ & 0.928 & & & \\
\hline $3 \mathrm{D}$ rotation angiography & 1.291 & $0.744-2.240$ & 0.363 & & & \\
\hline Interventional procedure & 2.394 & $1.339-4.282$ & 0.003 & 1.745 & $0.767-3.971$ & 0.185 \\
\hline Anti-coagulation therapy & 1.804 & $1.260-2.581$ & 0.01 & 1.469 & $0.557-3.874$ & 0.437 \\
\hline
\end{tabular}

3D: three-dimensional, CI: confidence interval.

Table 4 Univariate and multivariate analysis of risk factors in diagnostic cerebral angiography

\begin{tabular}{|c|c|c|c|c|c|c|}
\hline & \multicolumn{3}{|c|}{ Univariate analysis } & \multicolumn{3}{|c|}{ Multivariate analysis } \\
\hline Amount of contrast medium $\geq 100 \mathrm{ml}$ & 2.383 & $1.157-4.910$ & 0.019 & 2.038 & $0.840-4.943$ & 0.115 \\
\hline Number of examinations $\geq 10$ & 2.054 & $1.011-4.174$ & 0.047 & 1.599 & $0.665-3.841$ & 0.58 \\
\hline Resident operator & 2.417 & $1.185-4.929$ & 0.015 & 2.526 & $1.214-5.254$ & 0.013 \\
\hline
\end{tabular}

3D: three-dimensional, CI: confidence interval.

phy. ${ }^{10,12)}$ In our study, $72(29.1 \%)$ of 247 cerebral angiography procedures resulted in HIL on DWI. Age, past history of cerebral infarction, and previous anti-platelet therapy were significantly correlated with HIL on DWI. The findings demonstrate that arteriosclerosis or vasculopathy might be risk factors for incidental embolism after angiography. Patients with symptomatic carotid artery stenosis had a neurological complication rate as high as $10 \%{ }^{77}$ However, there was no significant difference between subsequent diseases in this study.

Previous articles have reported that patients who undergo neuro-interventional procedures carry a greater risk of presentation of HIL on DWI, which ranges from $51.3 \%$ to $61 \% .{ }^{17,18,21)}$ In the present study, HIL on DWI were detected after $31(43 \%)$ of 73 interventional procedures. Neuro-interventional procedures usually take longer, require more contrast medium, and involve increased procedural complexity, which could explain the higher risk of embolisms in an interventional procedure than in diagnostic cerebral angiography.

The use of higher amounts of contrast medium was also a risk factor for embolism in our study, although the total procedure time and the total num- ber of examinations were not significantly different. Evaluation of the frequency of embolic events during cerebral angiography by continuous transcranial Doppler monitoring found that embolic phenomena were detected in all procedure phases, but were more frequent during catheter flushing and injection of the contrast medium than during catheter and guide wire manipulation. ${ }^{6)}$ We speculate that the usage of contrast medium for checking vessels or catheter position may frequently cause embolic sources, such as blood clots and micro bubbles, during exchange from guide wire to syringe. The performance of diagnostic angiography by resident operators was significantly associated with HIL on DWI. The difference in experience might have affected the frequency of embolism. Therefore, we propose that additional angiography training should be provided to inexperienced operators, possibly using a simulator.

The present study has several limitations. Not all HIL might have been infarctions, because we did not perform DWI before angiography. This study was a retrospective study, so there will be substantial selective bias. Specifically, our university hospital treats no emergency patients. We need a prospective 
study to prove this result for future recommendations.

Cerebral embolism is not a rare complication of cerebral angiography. Age, past history of cerebral infarction, and previous anti-platelet therapies determine the risk for atherosclerotic changes in patients. Neuro-intervention and total amount of contrast medium determine the risk of time limitation for angiography. Moreover, the present study indicated that senior operators administered diagnostic cerebral angiography more safely than resident operators. This study indicates the necessity of lessening the risk of time limitations for angiography and the risk related to operator inexperience. As such, we recommend reducing the occurrence of embolic complications by providing further training. Furthermore, experience with angiography simulation training devices will help us to reduce the occurrence of procedural complications.

\section{Acknowledgment}

This work was partly supported by a Grant-in-Aid for Scientific Research (C), No.23592085 and the Ministry of Education, Culture, Sports, Science, and Technology-Japan.

Department of Biostatics in University of Tsukuba at Critical Path Research and Education Integrated Leading Center supported us in statistical analysis.

\section{Conflicts of Interest Disclosure}

We declare no conflicts of interest. All authors who are members of The Japan Neurosurgical Society (JNS) have registered online Self-reported COI Disclosure Statement Forms through the website for JNS members.

\section{References}

1) Bendszus $M$, Koltzenburg $M$, Burger R, WarmuthMetz M, Hofmann E, Solymosi L: Silent embolism in diagnostic cerebral angiography and neurointerventional procedures: a prospective study. Lancet 354: 1594-1597, 1999

2) Britt PM, Heiserman JE, Snider RM, Shill HA, Bird CR, Wallace RC: Incidence of postangiographic abnormalities revealed by diffusion-weighted MR imaging. AJNR Am J Neuroradiol 21: 55-59, 2000

3) Burdette JH, Ricci PE, Petitti N, Elster AD: Cerebral infarction: time course of signal intensity changes on diffusion-weighted MR images. AJR Am J Roentgenol 171: 791-795, 1998

4) Busing KA, Schulte-Sasse C, Fluchter S, Suselbeck T, Haase KK, Neff W, Hirsch JG, Borggrefe M, Duber C: Cerebral infarction: incidence and risk factors after diagnostic and interventional cardiac catheterization-prospective evaluation at diffusion-weighted MR imaging. Radiology 235: 177-183, 2005

5) Chuah KC, Stuckey SL, Berman IG: Silent embolism in diagnostic cerebral angiography: detection with diffusion-weighted imaging. Australas Radiol 48: 133-138, 2004

6) Dagirmanjian A, Davis DA, Rothfus WE, Deeb ZL, Goldberg AL: Silent cerebral microemboli occurring during carotid angiography: frequency as determined with Doppler sonography. AJR Am J Roentgenol 161: 1037-1040, 1993

7) Davies KN, Humphrey PR: Complications of cerebral angiography in patients with symptomatic carotid territory ischaemia screened by carotid ultrasound. J Neurol Neurosurg Psychiatry 56: 967-972, 1993

8) Dawkins AA, Evans AL, Wattam J, Romanowski CA, Connolly DJ, Hodgson TJ, Coley SC: Complications of cerebral angiography: a prospective analysis of 2,924 consecutive procedures. Neuroradiology 49: 753-759, 2007

9) Fifi JT, Meyers PM, Lavine SD, Cox V, Silverberg L, Mangla S, Pile-Spellman J: Complications of modern diagnostic cerebral angiography in an academic medical center. J Vasc Interv Radiol 20: 442-447, 2009

10) Hankey GJ, Warlow CP, Sellar RJ: Cerebral angiographic risk in mild cerebrovascular disease. Stroke 21: 209-222, 1990

11) Heiserman JE: Silent embolism after cerebral angiography-what harm? Lancet 354(9190): 15771578, 1999

12) Heiserman JE, Dean BL, Hodak JA, Flom RA, Bird CR, Drayer BP, Fram EK: Neurologic complications of cerebral angiography. AJNR Am J Neuroradiol 15: 1401-1411, 1994

13) Johnston DC, Chapman KM, Goldstein LB: Low rate of complications of cerebral angiography in routine clinical practice. Neurology 57: 2012-2014, 2001

14) Kato K, Tomura N, Takahashi S, Sakuma I, Watarai J: Ischemic lesions related to cerebral angiography: Evaluation by diffusion weighted MR imaging. Neuroradiology 45: 39-43, 2003

15) Kim IC, Hur SH, Park NH, Jun DH, Cho YK, Nam CW, Kim H, Han SW, Choi SY, Kim YN, Kim KB: Incidence and predictors of silent embolic cerebral infarction following diagnostic coronary angiography. Int J Cardiol 148: 179-182, 2011

16) Leonardi M, Cenni P, Simonetti L, Raffi L, Battaglia S: Retrospective study of complications arising during cerebral and spinal diagnostic angiography from 1998 to 2003. Interv Neuroradiol 11: 213-221, 2005

17) Rordorf G, Bellon RJ, Budzik RE Jr, Farkas J, Reinking GF, Pergolizzi RS, Ezzeddine M, Norbash AM, Gonzalez RG, Putman CM: Silent thromboembolic events associated with the treatment of unruptured cerebral aneurysms by use of Guglielmi detachable coils: prospective study applying diffusion-weighted imaging. AJNR Am J Neuroradiol 22: 5-10, 2001

18) Sakai H, Sakai N, Nakahara I, Shimozuru T, Higashi 
T, Takahashi JC, Ohta H, Kokuzawa J, Manaka H, Morizane A, Kawabata Y, Nagata I, Kikuchi H: Embolic complications of endovascular surgery for cerebrovascular diseases. Evaluation with diffusionweighted MR imaging. Interv Neuroradiol 6 Suppl 1: 223-226, 2000

19) Sasaki M, Ida M, Yamada K, Watanabe Y, Matsui M: Standardizing display conditions of diffusionweighted images using concurrent b0 images: a multi-vendor multi-institutional study. Magn Reson Med Sci 6: 133-137, 2007

20) Shibazaki K, Iguchi Y, Kimura K, Ueno Y, Inoue T: New asymptomatic ischemic lesions on diffusionweighted imaging after cerebral angiography. J Neurol Sci 266: 150-155, 2008

21) Soeda A, Sakai N, Sakai H, Iihara K, Yamada N, Imakita S, Nagata I: Thromboembolic events associated with Guglielmi detachable coil embolization of asymptomatic cerebral aneurysms: evaluation of 66 con- secutive cases with use of diffusion-weighted MR imaging. AJNR Am J Neuroradiol 24: 127-132, 2003

22) Sorensen AG, Buonanno FS, Gonzalez RG, Schwamm LH, Lev MH, Huang-Hellinger FR, Reese TG, Weisskoff RM, Davis TL, Suwanwela N, Can U, Moreira JA, Copen WA, Look RB, Finklestein SP, Rosen BR, Koroshetz WJ: Hyperacute stroke: evaluation with combined multisection diffusion-weighted and hemodynamically weighted echo-planar MR imaging. Radiology 199: 391-401, 1996

Address reprint requests to: Hideo Tsurushima, $\mathrm{MD}, \mathrm{PhD}$, Department of Neurosurgery, Faculty of Medicine, University of Tsukuba, 1-1-1 Tennodai, Tsukuba, Ibaraki 305-8575, Japan.

e-mail: hideo-tsurushima@md.tsukuba.ac.jp 